\title{
Malnutrition in the Twenty-First Century: An Epidemic Affecting Surgical Outcome
}

\author{
Jesus M. Culebras
}

$\mathbf{T}$ He RATE of malnourished PeOple among the general population has increased progressively during recent decades. This is especially evident among elderly patients. It has been known for more than a century that malnutrition is an ominous risk for surgical outcome. Dudley showed in 1936 that malnourished patients undergoing surgery for duodenal ulcer had a much higher death rate than those who were well nourished [1]. Many studies have documented the relationship between malnutrition and impaired host immune responses [2,3]. The association between poor pre-operative nutritional status and poor surgical outcome is evident. Malnutrition is associated with delayed recovery, higher rates of morbidity and mortality, prolonged hospital stay, and both increased healthcare cost and a higher early re-admission rate. These facts have a negative effect on hospitalized patients.

The Prague Declaration, signed in 2009 by representatives of the Ministries of Health of the European Union member states, the Czech presidency of the EU, medical experts, representatives of national health services and health insurance groups, the European Society of Parenteral and Enteral Nutrition, and the European Nutritional Health Alliance, arrived at the unanimous conclusion that malnutrition, including disease-related malnutrition, is an urgent public health and healthcare problem in Europe. They also stressed that suitable measures must be taken to prevent malnutrition because of its continuous compromising effect on patient quality of life, unnecessary morbidity and associated mortality, and the fact that it will continue to undermine the efficacy of European health services [4].

\section{Malnutrition and Infection}

Extensive studies have detailed the numerous risk factors associated with infection. One of them is malnutrition [5]. Malnutrition is associated with defects in immune function that may impair the host response to malignant disease [6]. The alterations in the host defense mechanism make patients highly susceptible to postoperative infections. Multiple components of the diet may affect immune function.

There is evidence that giving patients peri-operative nutritional supplements with immuno-nutritional additives can modulate favorably the immune and inflammatory response both in vitro and in patients with infection, trauma, burns, or those undergoing oncologic surgery [7]. In particular, the important role of amino acids, dietary nucleotides, and lipids in modulating immune function has been recognized $[8,9]$. Surgical site infections tend to be less frequent in the groups with enriched diets. Snyderman et al. [10] was interested to note that the increased number of infectious complications that were observed with standard formulas was mostly due to infections at distant sites (e.g., lungs, urinary tract) rather than surgical site infections. This finding implies that most surgical site infection and fistulas have different risk factors and may be attributable to surgical technique rather than depressed immune function.

\section{Malnutrition in the Elderly: Sarcopenia}

Demographic data are striking. The population of the world will increase by 2.6 billion in the next 40 years and population aging will result in a rapid increase of people ages 60 years and older. The consequence will be that the number of older persons (60 years and older) will triple and the oldest persons, that is, more than 80 years, of age will increase from 69 million to 377 million.

Malnutrition in older people is common, overlooked frequently, and results in many negative health outcomes. The absolute number of older people who are malnourished is increasing and many more are at risk of developing malnutrition [11]. Malnourished older people are also at increased risk of experiencing falls, prolonged hospitalization, institutionalization, post-operative complications and infections, pressure ulcers and complicated wound healing, and death.

Nutritional frailty refers to the disability that occurs in old age due to the unintentional physiological or pathological loss of body weight and sarcopenia. Sarcopenia is the decline in muscle mass and strength that may occur with healthy aging, and is believed to be both a process and an outcome. The anorexia of aging describes the physiologic decrease in appetite and food intake that accompanies normal aging that may result in undesirable weight loss. Compounding these physiologic effects are numerous socioeconomic and health (non-physiologic) causes of malnutrition. With aging, there is a decreased margin of 
homeostatic reserve and an increased likelihood of experiencing numerous assaults to homeostatic balance that in turn increase the risk of frailty. Social isolation leads to decreased nutrient intake.

\section{The Spanish Study about Hospital Malnutrition}

The Spanish Society for Parenteral and Enteral Nutrition aimed to provide the Spanish National Health System with relevant information about the prevalence of hospital malnutrition in Spain and how it is distributed among the different care areas, both at admission and discharge, as well as to estimate associated cost [12]. A nationwide, crosssectional, observational, multi-center study, in routine clinical practice, that evaluated the prevalence of hospital malnutrition using the NRS-2002 screening test both on patient admission and at discharge, was performed in 31 public hospitals by Planas et al. [13] and Alvarez et al. [14]. One thousand five hundred ninety seven patients had complete admission and discharge data and were analyzed. Fifty-four percent of all patients included were male, with a mean age of 63 years. Fifty-five percent of all patients included were $\geq 65$ years old. In $11.4 \%$ of all patients the body mass index (BMI) was below $18.5 \mathrm{~kg} / \mathrm{m}^{2}$. Thirtyseven percent of patients scored positive for malnutrition (NRS-2002 >3) and were considered to be malnourished, which represents $23 \%$ of all patients at admission. The prevalence of malnutrition in surgical patients was not significantly different than the general prevalence. When analyzing the prevalence of malnutrition according to age, gender, and type of hospital admission, the following points were observed: A significantly greater nutritional risk for elderly patients (age $\geq 70$ ) with respect to the rest $($ age $<70)$, women with respect to men, and those admitted as emergencies. Malnourished patients were, on average, 10 years older, had a mean weight of $58.5 \mathrm{~kg}$ (almost $13 \mathrm{~kg}$ less,) and a BMI four points lower than those who were not malnourished. Brachial and calf circumferences were significantly smaller than those of well-nourished patients. Clinical chemistry results showed the serum albumin concentration and lymphocyte counts to be significantly lower than in patients not at nutritional risk. Clinical conditions associated significantly at admission with a higher

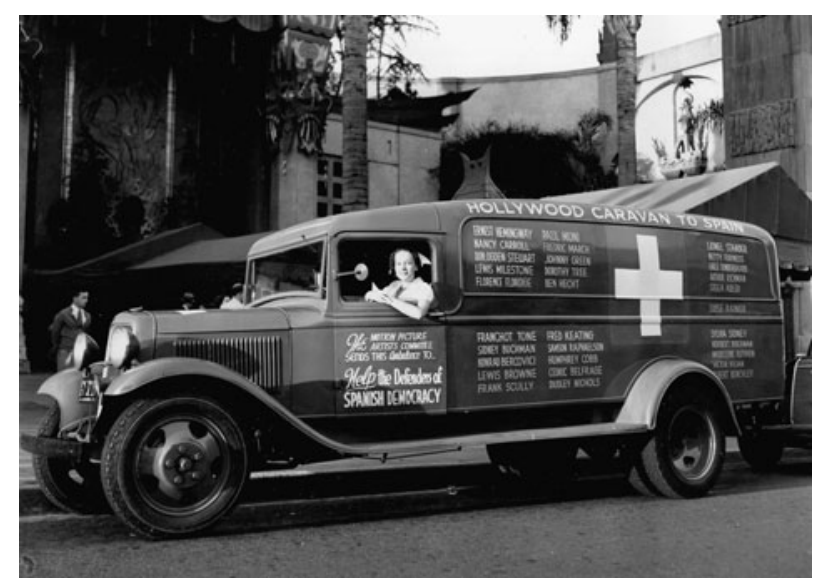

FIG. 1. Ambulance donated by the North American Committee to Aid Spanish Democracy. prevalence of malnutrition were dysphagia, neurological pathology, cancer, diabetes mellitus, and cardiovascular disease. A high prevalence of malnutrition was observed specifically in patients with degenerative neurologic diseases, (e.g., Alzheimer, Parkinson) and in patients with heart failure. Polymedicated patients had doubled the prevalence of malnutrition compared to non-polymedicated patients. Fourteen percent of all patients analyzed at discharge received some type of nutritional support during hospitalization, representing $29.5 \%$ of malnourished patients at discharge and $27.7 \%$ of all malnourished patients at admission. Nearly $10 \%$ of patients who showed no nutritional risk at admission developed malnutrition during hospitalization, and $72 \%$ of patients who were malnourished at admission remained malnourished at discharge. Mean hospital stay was significantly longer in malnourished patients compared with non-malnourished patients, at admission and at discharge. A clear relationship was observed between a change in the nutritional status during hospitalization and the length of stay, 8.0 5 5.2 days in patients who were admitted and remained non-malnourished compared with $15.2 \pm 9.2$ days in those non-malnourished at admission but who were malnourished at discharge.

Being age 70 or over, having a diagnosis of cancer or diabetes mellitus referral for dysphagia, and high consumption of medicines or alcohol correlated with a greater influence on a higher risk of malnutrition at admission. Mean hospital stay was significantly longer for patients with malnutrition compared with those without malnutrition. In economic terms, differences in length of stay translated into higher cost associated with patients malnourished at admission. The most outstanding difference was observed between those patients not presenting with malnutrition at any time and those who were non-malnourished at admission but were malnourished at discharge. This is especially relevant



FIG. 2. Doctor Antonio Culebras and his wife, Manuela, in the headquarters of Blood Transfusion in Vergara Street, Madrid, 1936. Culebras was chief commander of Blood Transfusion of the Armies of Extremadura and Andalucia. His wife was a volunteer nurse. 


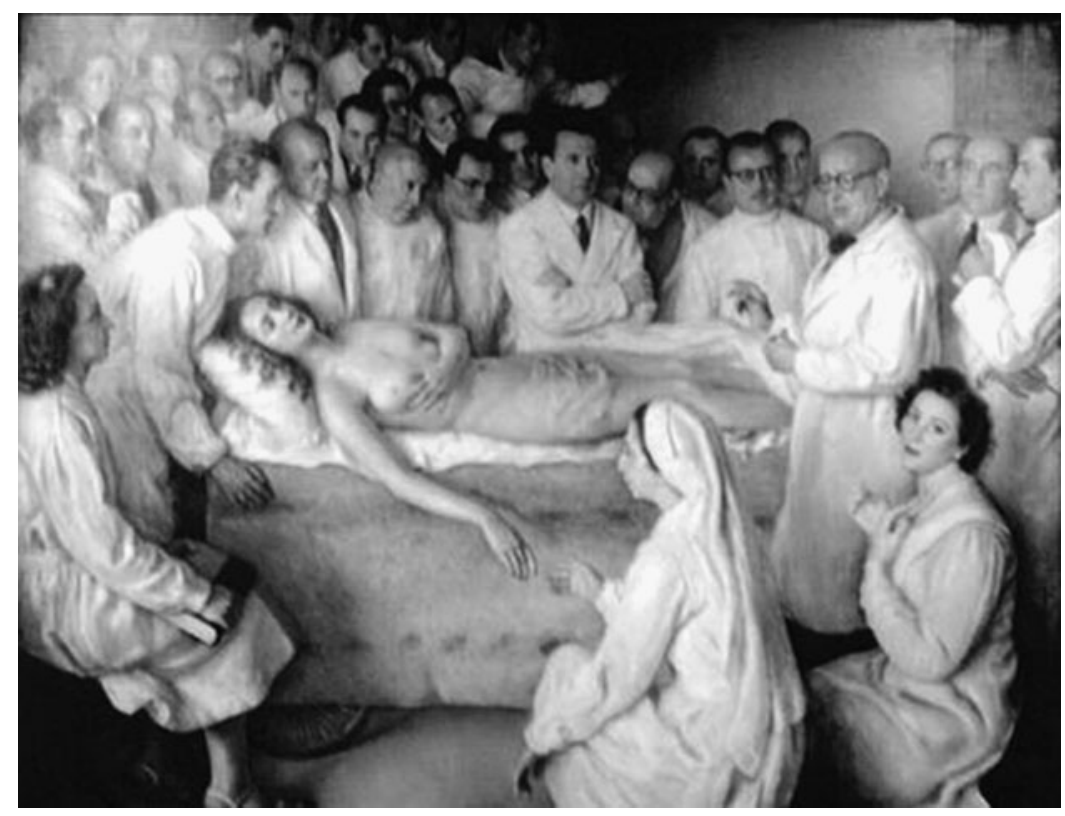

FIG. 3. Clinical session of Doctor Jimenez-Diaz. Oil on canvas. Eugenio Hermoso 1950. Fundación Jimenez Diaz, Madrid (details in the text).

considering that it affected nearly $10 \%$ of the study population and led to an extra cost of approximately $\$ 8,000$ per patient. The association of malnutrition and increased hospital cost is consistent with the results of other national and international studies [15-17].

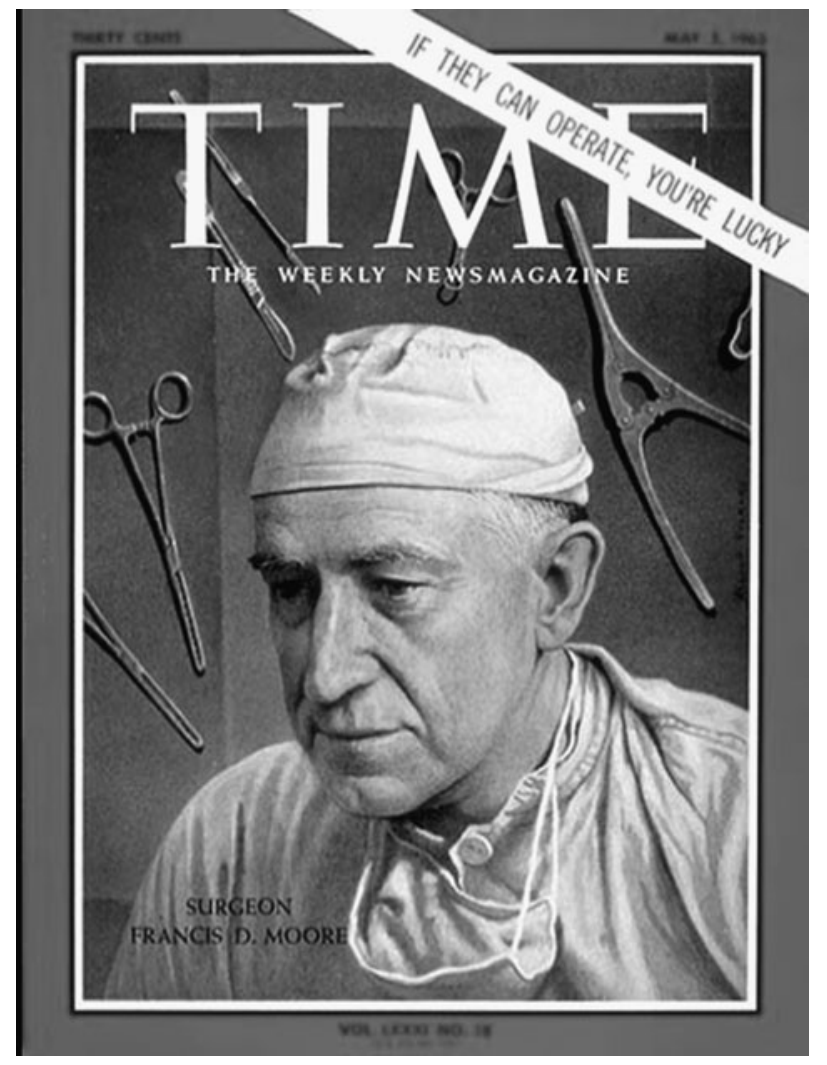

FIG. 4. Francis D. Moore appeared in the cover of Time magazine in 1963.
The above data by Alvarez et al. [14] confirm that hospital malnutrition prevalence is high in Spanish hospitals and even higher in patients more than 70 years old. Still more serious is the fact that overall prevalence remains unchanged at discharge, observing a slight decrease in patients age $\geq 70$ years. These results are consistent with similar recently published studies in Europe [18] and reveal that one of every four patients admitted to our hospitals is at risk of malnutrition, which is also associated with substantial extra cost for the health system. Patients' stratification according to medical or surgical wards showed no differences; therefore, these results also apply to surgical patients.

\section{Comments}

It is necessary to diagnose the degree of malnutrition and associate it with the need for nutritional therapy and its intensity, because outcome may be improved and resource consumption may be reduced (length of stay and other parameters). Main obstacles to improving nutritional care include lack of awareness for the problem among the general public and lack of awareness among decision makers and even care providers.

Weight loss exceeding $10 \%$ is an important and independent risk factor for complications, especially infection. Malnutrition, as reflected by weight loss, appears to be an independent factor for post-operative morbidity, even if adjusted for the effects of site of primary tumor, the consequent type of surgery, age, and serum albumins. The risk of complications increases concentration as the percent of weight loss exceeds 10\% [19].

Malnutrition is most often associated with pathologies requiring surgical intervention such as cancer or with the presence of dysphagia, and with medical situations that complicate surgery such as diabetes mellitus. Elderly and female surgical progressive patients have greater risk of malnutrition; emergency surgery is associated with a higher rate 


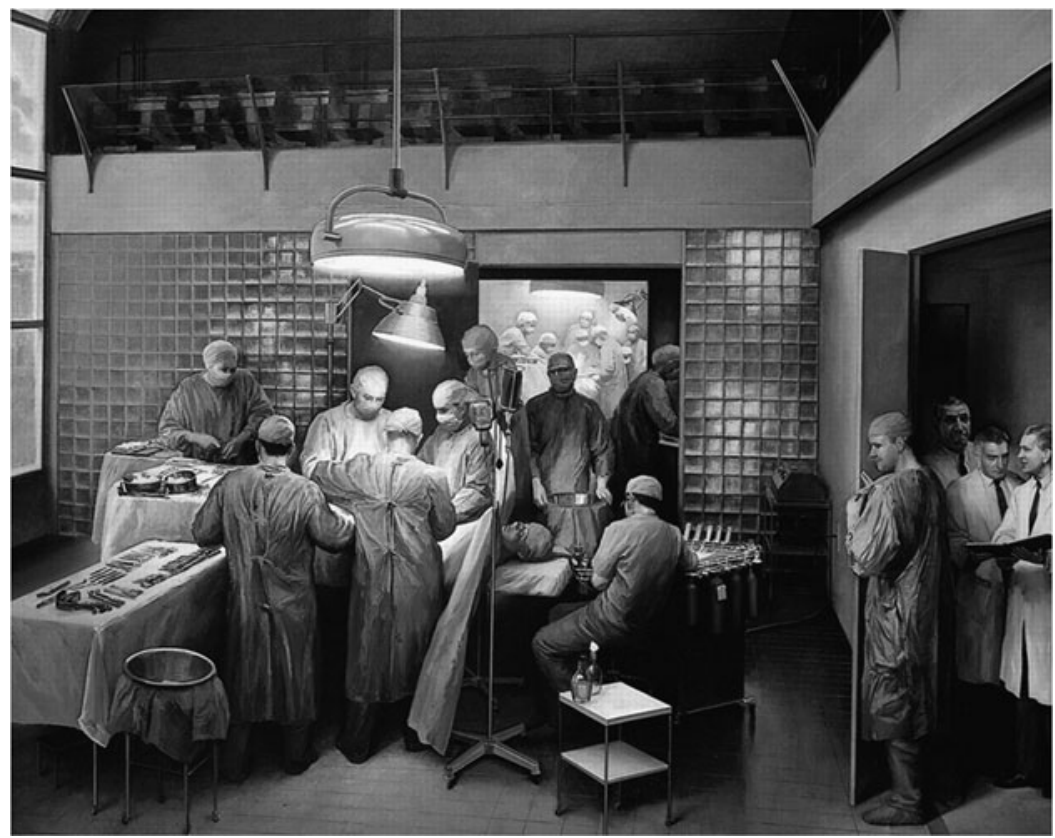

FIG. 5. The first successful kidney transplantation. Oil on canvas. Joel Babb 1995. The Countway Library, Boston (details in the text).

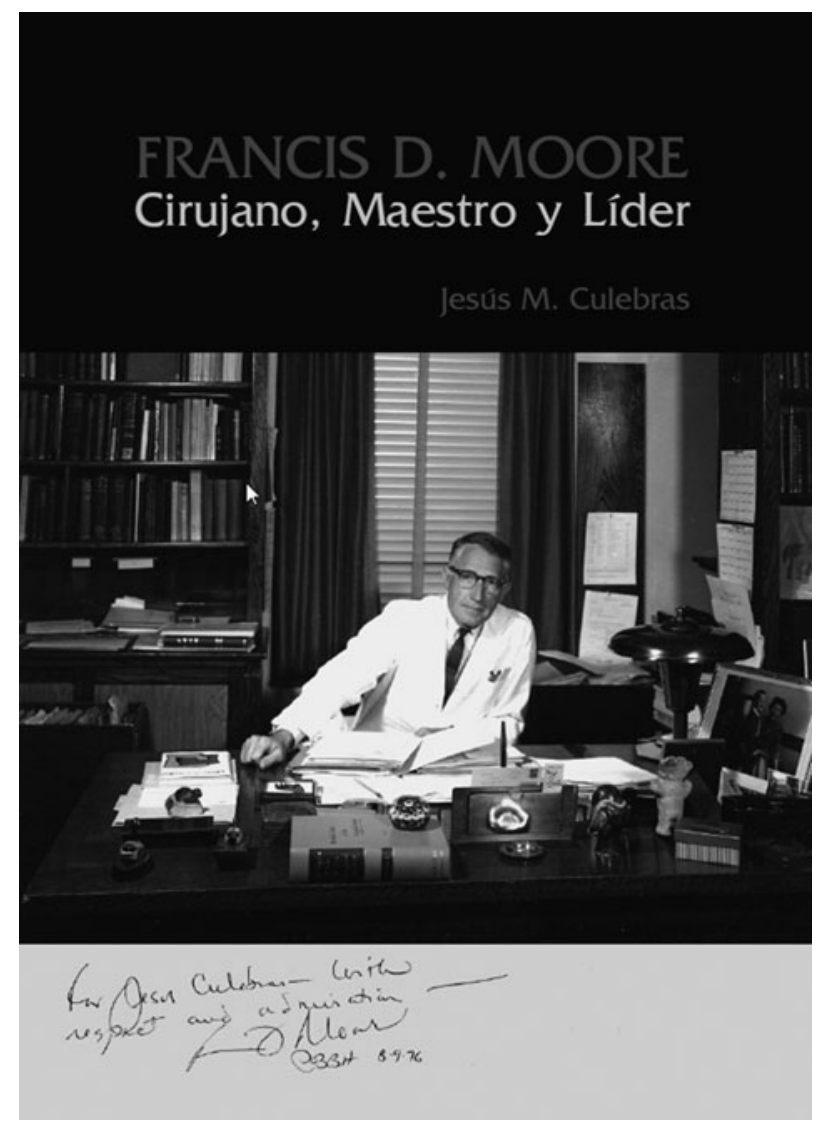

FIG. 6. Cover of the book Francis Moore: Surgeon, Teacher, and Leader. of malnutrition than scheduled surgery. Measures required for prevention and integrated care of the patient at risk of malnutrition or who is undernourished are simple, whereas the cost of a complicated surgical outcome, with infection, fistulae, or wound dehiscence, is much higher, both in economic terms and compared with the course and outcome of the disease.

Nutritional interventions as oral nutritional supplementation, in surgical hospitalized patients at medium- or high risk of malnutrition, are associated with reductions in infection rate, length of hospital stay, reduced bed-day cost and complication cost as compared with patients who receive no nutritional supplementation. Thorough knowledge and interest in patients' nutritional status by the healthcare staff should always be promoted. Strategies to avoid periods of fasting and to identify patients at risk of malnutrition should be implemented. Considering the high prevalence of malnutrition and its repercussions in patient morbidity-mortality and healthcare cost, nutritional screening measures must be included in an integrated nutritional care plan for patients while in the hospital.

Early and periodic nutritional assessment must be carried out in order to identify malnourished patients in clinical practice who, if not given the right care, will be at greater risk of suffering disease- or treatment-related complications. It is also necessary to diagnose the degree of malnutrition and associate it with the need for nutritional therapy and its intensity, because outcome may be improved and resource consumption may be reduced.

Action points should include public awareness and education, guideline development and implementation, mandatory screening, the promotion and support of research on malnutrition, training in nutritional care among caregivers, and the establishment of nutritional care plans supported by the authorities. Governments and institutions should be 


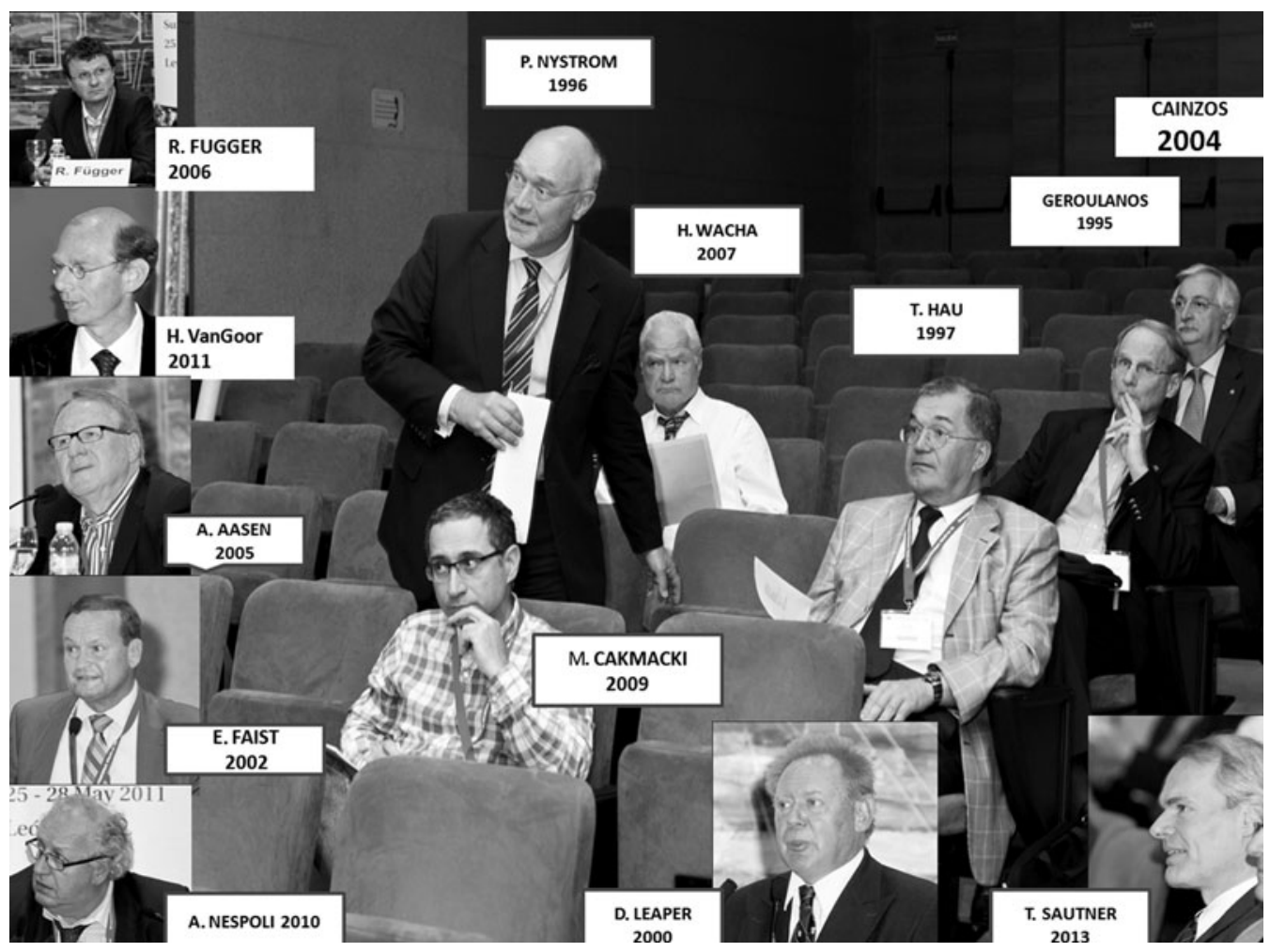

FIG. 7. Surgical Infection Society-Europe past presidents at the 24th European Congress of the Society in Leon, May 26-28, 2011.

forced to intervene in malnutrition, which is Europe's hidden major health problem.

\section{Other Personal Considerations from the President of the Surgical Infection Society-Europe}

I have been lucky in several phases of my professional life. The familial environment provided by my parents created an intellectual atmosphere that marked my future career. My father, Antonio Culebras, was a physician who, during the
Spanish civil war (1936-39), had an important participation in organizing the blood transfusion teams that served in the operating theaters of the battlefields on the side of the legally elected government of the Spanish Republic.

Shortly after the outbreak of civil war in Spain in 1936, Edward K. Barsky, from New York, led the North American Committee to Aid Spanish Democracy. A team of American and Canadian doctors, nurses, and technicians went to Spain with the Abraham Lincoln Brigade from the United States and the Mackencie-Pappineau Battalion from

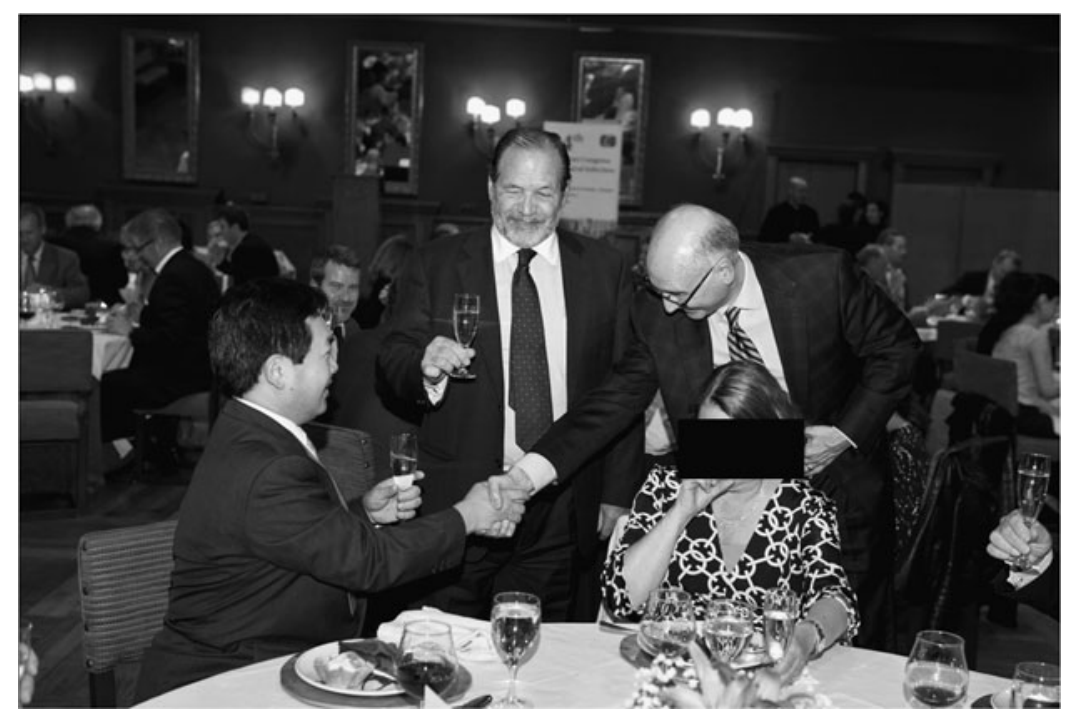

FIG. 8. Three sister societies met at the 24th SIS-E Congress, Leon, 2011. From left to right: Yuichi Yoshida (Japanese Surgical Infection Society), Jesus Culebras (SIS-E), and John Alverdy (Surgical Infection Society-North America). 
Canada. Ambulances and other medical equipment and supplies were donated from the United States, Canada, France, UK, and other countries (Fig. 1).

A Canadian surgeon, Norman Bethune, from Ontario, also came to Spain at that time. Barsky, Bethune, and Culebras organized base hospitals and convalescent homes in schools and monasteries. Emergency surgery units were established at the front in ambulances and makeshift tents. Mobile medical hospitals outfitted with battery-powered operating rooms were distributed in the rear areas. The units contained dressings for 500 wounds, and enough supplies and medicine for 100 operations.

The idea of administering blood transfusions on the spot was developed. They organized a service to collect blood from donors and deliver it to the battlefront, thereby saving countless lives. Many of the techniques applied in the Spanish front were later exported to World War II. The mobile medical units devised by Barsky, Bethune, and Culebras were precursors to the later development of Mobile Army Surgical Hospital units (Fig. 2).

After the war Doctor Antonio Culebras was convicted, imprisoned, and separated from the university [20]. Dr. A. Culebras died tragically in an automobile accident in 1954.

I was lucky in my academic post-graduate training. Both during my student years and during my surgical residency I was trained in the Fundacion Jimenez Diaz. This university hospital was founded by Professor Jimenez Diaz in 1953, being the first modern hospital in Spain. It was in this hospital where the first Spanish intensive care unit was created and operations under extracorporeal circulation were performed for the first time in Spain.

The painting by Hermoso (Fig. 3) represents Jimenez Diaz surrounded by his coworkers and pupils while exploring a young woman. I had the opportunity to learn from many of the physicians and surgeons represented in the painting.

I was lucky to continue my surgical training at the Peter Bent Brigham Hospital (PBBH) with Francis D. Moore (Fig. 4). Moore is perhaps the most important American surgeon of the second half of the 20th century. Moore was a pioneer in numerous experimental surgical treatments. More than 1,000 surgeons have been trained at the PBBH (now Brigham and Womens Hospital) during the 30-year period in which Moore was Moseley Professor of Surgery and surgeon-in-chief of the Department of Surgery. The "Brigham" pioneered and continues performing, many important innovations in medicine. Kidney transplantation, liver transplantation, heart surgery, surgical metabolism, body composition, and parenteral nutrition have had great contributions from the "Brigham."

The picture in Figure 5 (oil on canvas $70 \times 88$ in by Joel Babb, painted in 1995) shows the first kidney transplant between identical twins. The event took place in 1954. The surgeon implanting the kidney is Joseph Murray, assisted by John Robotham. The anesthesist is Leroy Vandam. Francis Moore appears in the center of the painting. He is "the surgical assistant" who carries the kidney from the donor to the recipient. On the righthand side, still with surgical gown but without the mask, is Hartwell Harrison, the donor surgeon. Behind him is Gustave Dammin, pathologist; John P. Merrill, physician-nephrologist; and George W. Thorn, physician-in-chief. These three figures represent the underlying science and wisdom of medicine.

Every transplant done today is, in a real sense, a direct outgrowth of this first venture. Joseph Murray received the
Nobel Prize in 1991 [21]. After finishing my three-year training at the "Brigham," Moore and I became friends, staying in contact until his death in 2001. I wrote a book about Francis Moore in 2008 [22] (Fig. 6). The book is accessible at www.nutricionhospitalaria.com/LIBRO_FRANCIS_MOORE .pdf.

I was lucky to get in touch with the Surgical Infection Society of Europe in 1989, a couple of years after its foundation. Professor Cainzos, one of the SIS-E founders, invited me to join the Spanish National Committteee of Surgical Infections from which we cooperated closely with SIS-E [23]. After that invitation I had the chance to serve the SIS-E Council in different positions during a long period of time. I had the opportunity to meet and work with many SIS-E members. I was honored by SIS-E with the commitment to organize the 24th Congress in Leon, the town where I work and live. The zenith of my cooperation with SIS-E is presented in Figure 7: Thirteen past presidents of SIS-E attended the Congress in Leon; from left to right in the photograph: Reinhold Függer, Austria, 2006; Harry van Goor, The Netherlands, 2011; Ansgar Aasen, Norway, 2003; Eugen Faist, Germany, 2002; Angelo Nespoli, Italy, 2010; Metin Cakmacki, Turkey, 2009; Per-Olof Nyström, Sweden, 1996; Hannes Wacha, Germany, 2007; David Leaper, UK, 2000; Toni Hau, Germany, 1997; Stefanos Geroulanos, Greece, 1995; and Miguel Cainzos, Spain, 2004. Thomas Sautner, from Austria, is the 2013 president. Roland Andersson, from Lund, Sweden, was supposed to be there also but an ash cloud prevented him from travelling. The extensive presence of past SIS-E presidents is a clear indicator of how strong the links are among our members.

There was representation also from our sister societies from North America and Japan (Fig. 8). The recently created Surgical Infection Society of Latin America (SIS-LA) is also planning to cooperate in the future.

The SIS-E was founded a quarter of a century ago. Many achievements have been obtained during these 25 years. I foresee that many more will be obtained in the forthcoming years.

\section{References}

1. Studley HO. Percentage of weight loss. A basic indicator of surgical risk in patients with chronic peptic ulcer. JAMA 1936;106:458-460.

2. Mainous MR, Deitch E. Nutrition and infection. Surg Clin North Am 1994;74:659-676.

3. Waitzberg DL, Ravacci GR, Raslan M. Hospital hyponutrition. Nutr Hosp 2011;26:254-264.

4. The Prague declaration: Stop disease-related malnutrition. Available at www.european-nutrition. org/files/pdf_pdf 66.pdf. Accessed November 25, 2010.

5. Fry DE. Fifty-ways to cause surgical site infections. Surg Infect 2011;12:497-500.

6. Culebras-Fernandez JM, De Paz Arias R, Jorquera-Plaza F, Garcia de Lorenzo A. Nutrition in the surgical patient: Immunonutrition. Nutr Hosp 2001;16:67-77.

7. Di carlo V, Gianotti I, Balzano G, et al. Complications of pancreatic surgery and the role of perioperative nutrition. Dig Dis Sci 1999;16:320-326.

8. Pérez de la Cruz AJ, Abilés J, Pérez Abud R. Perspectives in the design and development of new products for enteral nutrition. Nutr Hosp 2006;21(Suppl 2):98-108.

9. Casas-Rodera P, Gómez-Candela C, Benítez S, et al. Immunoenhanced enteral nutrition formulas in head and neck 
cancer surgery: A prospective, randomized clinical trial. Nutr Hosp 2008;23:105-110.

10. Snyderman $\mathrm{CH}$, Kachman K, Molseed L, et al. Reduced postoperative infections with immune-enhancing nutritional supplements. Laryngosc 1999;109:915-921.

11. Gómez-Cabello A., Vicente Rodríguez G., Vila-Maldonado S., et al. Aging and body composition: The sarcopenic obesity in Spain. Nutr Hosp 2012;27:22-30.

12. García de Lorenzo A, Álvarez Hernandez J, Planas M, et al. Multidisciplinary consensus on the approach to hospital malnutrition in Spain. Nutr Hosp 2011;26:701-710.

13. Planas Vila M, Álvarez Hernández J, García de Lorenzo A, et al. The burden of hospital malnutrition in Spain: Methods and development of the PREDyCES ${ }^{\circledR}$ study. Nutr Hosp 2010;25:1020-1024.

14. Álvarez-Hernández J, Planas Vila M, León-Sanz M, et al. Prevalence and costs of malnutrition in hospitalized patients; the PREDyCES ${ }^{\circledR}$ Study. Nutr Hosp 2012;27:10491059.

15. Lobo Támer G, Ruiz López MD, Pérez de la Cruz AJ. Hospital Malnutrition: Relation between the hospital length of stay and the rate of early readmissions. Med Clin (Barc) 2009;132:377-384.
16. De Ulibarri Perez JI, Picon Cesar MJ, Garcia Benavent E, Mancha Alvarez-Estrada A. Early detection and control of hospital malnutrition. Nutr Hosp 2002;17:139-146.

17. Pérez de la Cruz A, Lobo Tamer G, Orduna Espinosa R, et al. Malnutrition in hospitalized patients: Prevalence and economic impact. Med Clin (Barc) 2004;123:201-206.

18. Ljungqvist, $\mathrm{O}$, de Man, F. Under nutrition-a major health problem in Europe. Nutr Hosp 2009;24:368-370.

19. Bozzetti F, Gianotti L, Braga M, et al. Postoperative complications in gastrointestinal cancer patients: The joint role of the nutritional status and the nutritional support. Clin Nutr 2007;26:698-709.

20. Otero Carvajal E, Nuñez M, Gomez-Bravo G, et al. Destruction of science in Spain. University depuration during the Franco era. Edit Com 2006:83-113.

21. Nespoli A, Gerovlanos S, Nardone A, et al. Surg Infect 2011;12:3-13.

22. Culebras JM. Francis Moore, surgeon, teacher and leader. Ed. Sever-Cuesta, 2008, Valladolid, Spain. Available at www.nutricionhospitalaria.com/LIBRO_FRANCIS_MOORE.pdf

23. Cainzos M. Surgical Infection Society-Europe Presidential Address: Surgical Infection Control. Surg Infect 2005; 6(1):7-17. 